Www.jmscr.igmpublication.org

Index Copernicus Value: 79.54

ISSN (e)-2347-176x ISSN (p) 2455-0450

crossref DOI: https://dx.doi.org/10.18535/jmscr/v7i6.162

\title{
Incidence of Dry eye after clear corneal phacoemulsification
}

\author{
Authors \\ Dr Shazia Qayum ${ }^{*}$, Dr Asaf Iqbal ${ }^{2}$ \\ ${ }^{1}$ MS Ophthalmology DNB Ophthalmology, Assistant Professor, GMC \& AH Rajouri \\ ${ }^{2}$ Consultant Ophthalmologist, Rajouri \\ *Corresponding Author \\ Dr Shazia Qayum
}

MS ophthalmology DNB ophthalmology, Assistant Professor, GMC \& AH Rajouri, India

\begin{abstract}
Introduction: Dry eye syndrome is not a disease entity, but a symptom complex which occur as result of deficiency or abnormalities of tear film, exposing the conjunctival and corneal epithelium to evaporation. Corneal surgery has been recognized as one of the risk factors for the development of dry eye disease by interruption of normal ocular homeostasis by reducing corneal sensation and altering the contour of the ocular surface as a consequence of the inflammation caused by surgical trauma.

Objective: The aim of this study is to evaluate incidence of dry eye in patients who undergo cataract surgery by clear corneal phacoemulsification.

Methodology: A prospective study was conducted in the department of Ophthalmology at MMIMSR, Mullana from Jan 2018 to June 2018. A total of 100 patients were enrolled in the study. All the patients were subjected to Schirmer's I test (SIT), Rose bengal staining and tear film break up time (TBUT) examination and OSDI questionnaire. The dry eye incidence was analysed on 1,7,30 and 90 postoperative day.

Results: All the patients were subjected to Schirmer's I test (SIT), Rose bengal staining and tear film break up time (TBUT) examination and OSDI questionnaire. The dry eye incidence was analysed on 1,7,30 and 90 postoperative day. At $90^{\text {th }}$ postoperative day, 82 patients had normal TBUT and Schirmerl test while 18 patients showed abnormal TBUT and Schirmer1 test indicating an incidence of $18 \%$ of dry eye after clear corneal phacoemulsification.
\end{abstract}

Keywords: Dry eye, TBUT, Schirmer test, phacoemulsification, Rose Bengal Staining.

\section{Introduction}

Dry eye syndrome is not a disease entity, but a symptom complex which occur as result of deficiency or abnormalities of tear film, exposing the conjunctival and corneal epithelium to evaporation. Dry eye disease is a multifactorial ailment characterized by dryness of the ocular surface due to tear deficit and evaporation. ${ }^{1,2}$ There are multiple factors causing dry eye which include age, female gender, diabetes mellitus, use 
of contact lens, connective tissue disorder, drugs like anticholinergics, oral contraceptives, antidepressants, antihistamines and topical eye drops containing preservatives and ocular diseases like blepharitis, chronic conjunctivitis, meibomitis and pterygium. $^{3-5}$ The prevalence of dry eyes varies from $4 \%$ to $57 \%$ worldwide. $^{6-9}$

The International Dry Eye Workshop defined dry eye disease as "a multifactorial disease of the tears and ocular surface that results in symptoms of discomfort, visual disturbance, and tear film instability with potential damage to the ocular surface. It is accompanied by increased osmolarity of the tear film and inflammation of the ocular surface." 1

Corneal surgery has been recognized as one of the risk factors for the development of dry eye disease by interruption of normal ocular homeostasis by reducing corneal sensation and altering the contour of the ocular surface as a consequence of the inflammation caused by surgical trauma. ${ }^{10-}$ ${ }^{12}$ Clear corneal phacoemulsification has been turned out to be one of the safest, most successful, and regularly performed surgeries these days. As noticed with other corneal surgeries, it may modify the ocular surface and disturb the normal tear function. In a study done by Liu, reduction in tear secretion after phacoemulsification was noticed in diabetic patients. ${ }^{13}$

The aim of this study is to evaluate incidence of dry eye in patients who undergo cataract surgery by clear corneal phacoemulsification. We correlated the outcomes of various tear function tests, such as Schirmer test, fluoroscein tear film break-up time (FTBUT), conjunctival rose bengal staining and Ocular surface disease index (OSDI) questionnaire in various age groups of senile cataract with no preoperative dry eye.

\section{Methods}

A prospective study was conducted in the department of Ophthalmology at MMIMSR, Mullana from Jan 2018 to June 2018.100 patients admitted for phacoemulsification surgery were enrolled for the study after taking written consent.
We included the patients above 18 years of age. We excluded patients below 18 years of age, history of previous ocular surgery or laser, previous history of ocular trauma, chemical burns, contact lens usage and any history of drug intake which interfere with tear film abnormality. All patients underwent detailed pre- operative examination with slit lamp biomicroscopy and fundoscopy. All the patients were subjected to Schirmer's I test (SIT), Rose bengal staining and tear film break up time (TBUT) examination and OSDI questionnaire. The dry eye incidence was analysed on 1,7,30 and 90 postoperative day.

Schirmer's Test-I was done using $5 \mathrm{~mm}$ x $35 \mathrm{~mm}$ Whatman's filter paper 41 without topical anaesthetic drops. Schirmer's strip was folded 5 $\mathrm{mm}$ from one end and was inserted at the junction of inner $2 / 3$ and the outer $1 / 3$ and of lower lid margin. The patient was allowed to blink as necessary. The amount of wetting was measured after 5 minutes. The reading was interpreted as:

10 -- $15 \mathrm{~mm}$ : Mild dry eye

5 -- $10 \mathrm{~mm}$ : Moderate dry eye

$<5 \mathrm{~mm}$ : Severe dry eye

Rose Bengal Staining of the conjunctiva was achieved by using Rose Bengal paper strip, on the slit lamp using red free filter to perceive any staining of conjunctiva or cornea. Grading was done by separating the ocular surface into three zones: nasal bulbar conjunctiva, temporal bulbar conjunctiva and cornea, each graded 0-3 (0, none; 3 , confluent staining). Scores above 3 were measured abnormal and suggestive of dry eye.

Fluorescein Tear Film Break Up Time: was measured after introducing fluorescein dye into the inferior conjunctival fornix of patient and ask the patient to blink numerous times before stopping. The tear film was observed on a slit lamp with a broad beam of cobalt blue light for appearance of black spots indicating areas of dryness. The time interval between the last blink and the appearance of first dry spot was taken as breakup time. A breakup time of less than 10 seconds was considered abnormal. 


\section{Ocular Surface Disease Index (OSDI)} questionnaire: consists of 12 questions which were asked to the patients and scored according to the response of the patient. The OSDI is evaluated on a scale of 0 to 100 with higher scores meaning greater debility. Scores from 0 to 25 were considered normal; scores more than 25 designate the presence of dry eye symptoms.

\section{Results}

This study included 100 patients requiring cataract surgery presenting to MMIMSR, Mullana. They were randomly selected for clear corneal phacoemulsification technique with posterior chamber IOL implantation. A detailed preoperative examination was done. Incidence of dry eye in the patients was evaluated post operatively at day 1 , day 7 , day 30 and day 90 , with the help of various tests.

A total of 100 patients were enrolled during the study period of 6 months. The mean age of 58.03 years was reported with 36- 75 years range. More than half $(54 \%)$ of the patients belongs to age group of 51- 65 years. Female cases were more $(57 \%)$ than male. Majority of patients $(78.0 \%)$ were residing in urban areas. (Table no.1).

On preoperative examination, the mean of Schirmer I test was 25.36 with standard error of 0.496. On $1^{\text {st }}$ postoperative day, the mean of Schirmer I test was 24.48 with standard error of 0.635. On $7^{\text {th }}$ postoperative day, the mean of Schirmer I was 22.87 with standard error of 0.789 . On $30^{\text {th }}$ postoperative day, the mean value was 24.78 with standard error of 0.823 . On $90^{\text {th }}$ postoperative day, the mean was 25.11 with standard error of 0.692 .

The mean value of Rose Bengal staining scores was 0.42 preoperatively with standard error of mean as 0.06 . On day 1 postoperative, the mean was 0.65 with standard error of 0.17 . On $7^{\text {th }}$ day postoperatively, the mean value was 0.82 and standard error was 0.234 . On day 30 ,

the mean value was 0.79 with standard error of 0.173 . On $90^{\text {th }}$ postoperative day, the mean value of Rose Bengal staining scores was 0.69 with standard error of 0.122 . The pattern of Rose Bengal staining depicts that on day 7 , patients had confluent staining with increased no. of patients having higher scores which goes on decreasing with time.

On preoperative examination, the mean of Tear Film Break Up Time scores was 14.32 with standard error of 0.150 . On day 1 , the mean was 13.06 with standard error of mean as 0.274 . On day 7 , the mean was 11.75 and standard error was 0.332. On day 30 , the mean was 12.21 with standard error of 0.260 . On day 90, the mean of tear film break up scores was 12.89 with standard error of 0.234 . The pattern of tear film break up scores depicts that dry eye patients peaked at day 7 and the subsequent scores steadily enhanced over follow up days.

The mean of Ocular Surface Disease Index scores was 13.43 preoperatively with standard error of 0.42 . On day 1 , the mean was 13.43 with standard error of 0.42 . On day 7, the mean was 25.98 and standard error was 2.27 . On day 30 , the mean was 22.14 with standard error of 1.76 . On day 90 , the mean of OSDI score was 18.16 with standard error of 1.66. According to pattern of Ocular Surface Disease Index scores, dry eye patients peaked at day 7 and scores remained nearly the same on follow up.

At $90^{\text {th }}$ postoperative day, 82 patients had normal TBUT and Schirmer1 test while 18 patients showed abnormal TBUT and Schirmer1 test indicating an incidence of $18 \%$ of dry eye after clear corneal phacoemulsification.

Table No.1 Demographic data of the patients undergoing phacoemulsification

\begin{tabular}{|l|c|c|}
\hline \multicolumn{1}{|c|}{ Characteristics } & Number(n=100) & \%age \\
\hline Age Group & 18 & \\
<50 years & 54 & $18 \%$ \\
51-65 years & 28 & $28 \%$ \\
$>65$ years & & \\
\hline Sex & 43 & $43 \%$ \\
Male & 57 & $57 \%$ \\
female & 22 & \\
\hline Residential status & 78 & $78 \%$ \\
$\quad$ Rural & & \\
Urban & &
\end{tabular}


Table No. 2 Comparison of preoperative and postoperative TBUT, Schirmer 1 test, Rose Bengal staining and Ocular surface disease index questionnaire

\begin{tabular}{|l|c|c|}
\hline Variable & Mean value & $\begin{array}{c}\text { Standard error } \\
\text { 0f mean }\end{array}$ \\
\hline $\begin{array}{l}\text { TBUT } \\
\text { Preoperative } \\
\text { Postoperative } \\
\text { Day 1 }\end{array}$ & 14.32 & 0.150 \\
Day 7 & 13.06 & 0.274 \\
Day 30 & 11.75 & 0.332 \\
Day 90 & 12.21 & 0.260 \\
12.89 & 0.234 \\
\hline Schirmer 1 test & & \\
Preoperative & 25.36 & 0.496 \\
Postoperative & & \\
Day 1 & 24.48 & 0.635 \\
Day 7 & 22.87 & 0.789 \\
Day 30 & 24.78 & 0.823 \\
Day 90 & 25.11 & 0.692 \\
\hline Rose Bengal staining & & \\
Preoperative & 0.42 & 0.06 \\
Postoperative & & \\
Day 1 & 0.65 & 0.17 \\
Day 7 & 0.82 & 0.234 \\
Day 30 & 0.79 & 0.172 \\
Day 90 & 0.69 & 0.122 \\
\hline OSDI questionnaire & & \\
Preoperative & 13.43 & 0.42 \\
Postoperative & & \\
Day 1 & 13.43 & 0.42 \\
Day 7 & 25.98 & 2.27 \\
Day 30 & 22.14 & 1.76 \\
Day 90 & 18.16 & 1.66 \\
\hline
\end{tabular}

\section{Discussion}

Dry eye can occur after numerous types of ocular surgical procedures such as photorefractive keratectomy and laser assisted in situ keratomileusis (LASIK). Dry eye may continue for up to 6 months or more after LASIK with an incidence of $20 \% .^{3}$ According to annual report 2010-2011 of NPCB, cataract surgeries have significantly increased from 16 lakh in 1992-93 to 59.1 lakh in 2009-10. ${ }^{14}$ Numerous factors might disturb the ocular surface milieu after cataract surgery with utmost importance is corneal desensitization. $^{15}$

The present study was conducted on 100 patients attending the outpatient department with cataract and were planned for cataract surgery using clear corneal Phacoemulsification technique. All the tests performed preoperatively and postoperatively exhibited incidence of dry eye to be $11 \%$. Dry eye symptoms were maximum at day 7 in our study. The cornea is one of the most vastly innervated organs, with around 44 corneal nerve bundles entering the cornea around the limbus centripetally ${ }^{16}$ and larger nerve fibers that run from the 9 o'clock to the 3 o'clock position and divide to accomplish a homogenous supply over the entire cornea. ${ }^{17}$ Thus, it is susceptible to any injury within that region. Temporal corneal incisions made during phacoemulsification can diminish the corneal sensitivity in the operating area as well as areas far from the surgical site. ${ }^{18}$ The harm to the corneal nerves may increase when extended phacoemulsification time is required to break up a dense cataract. ${ }^{18}$ The corneal incisions may lead to neurogenic inflammation and various inflammatory mediators may diminish corneal sensitivity. ${ }^{19}$ Postoperatively as the cornea heals, neural growth factors are produced for the regeneration of the subepithelial axons of the cornea. $^{19}$

Therefore, the recovery of the nerves of the cornea may elucidate the occurrence of dry eye immediately after surgery and enhanced results were noticed in different tests performed afterwards in all test scores over 90 days. However, corneal nerves transections and damage to the corneal epithelium, prolonged exposure to light of operating microscope, intra operative irrigation of the tear film, use of topical eye drops given postoperatively and its preservatives can cause dry eye following phacoemulsification. ${ }^{13,20}$ Benzalkonium chloride is one of the most regularly used preservatives in topical eye drops which can induce tear film instability and reduces the number of mucin-producing cells. Additional factors linked with dry eye are older age, female gender, diabetes and systemic hypertension. However, in our study, dry eye was not associated with these factors because of small sample size in the study. Even though mild to moderate dry eye may not hinder vision, diminution of vision can occur in severe dry eye cases. 


\section{Conclusion}

This study exhibited that cataract surgery can cause or worsen dry eye therefore it is mandatory to do detailed examination of the patient preoperatively and patients should be informed about the probable exacerbation of dry eye symptoms after cataract surgery and artificial tears must be prescribed for the dry eye symptoms.

\section{References}

1. Lemp MA, Baudouin C, Baum J, et al. The definition and classification of dry eye disease: report of the Definition and Classification Subcommittee of the International Dry Eye Workshop. Ocular Surf. 2007;5:75-92.

2. Apostol S, Filip M, Dragne C, Filip A. Dry eye syndrome; etiological and therapeutic aspects. Oftalmologia. 2003;59:28-31.

3. Shoja MR, Besharati MR. Dry eye after LASIK for myopia: incidence and risk factors. Eur J Ophthalmol. 2007;17:1-6.

4. Moss SE, Klein R, Klein BE. Long-term incidence of dry eye in an older population. Optom Vis Sci. 2008;85:66874.

5. De Paiva CS, Chen Z, Koch DD, Hamill MB, Manuel FK, Hassan SS et al. The incidence and risk factors for developing dry eye after myopic LASIK. Am J Ophthalmol. 2006;141:438-45.

6. Murube J, Wilson S, Ramos-Esteban J. New horizons in the relief and control of dry eye. Highlights Opthalmol. 2001;26:55-64.

7. Schaumberg DA, Sullivan DA, Buring JE, Dana MR. Prevalence of dry eye syndrome among US women. Am J Ophthalmol. 2003;136:318-26.

8. Moss SE, Klein R, Klein BE. Prevalence of and risk factors for dry eye syndrome. Arch Ophthalmol. 2000;118:1264-8.

9. Schaumberg DA, Dana R, Buring JE, Sullivan DA. Prevalence of dry eye disease among US men: Estimates from the Physicians' Health Studies. Arch Ophthalmol. 2009;127:763-8.

10. Benitez-del-Castillo JM, Rio DT, Iradier T et al. Decrease in tear secretion and corneal sensitivity after laser-in-situ keratomileusis. Cornea .2001;20: 30-2.

11. Nassaralla BA, McLeod SD, Nassaralla JJ Jr. Effect of myopic LASIK on human corneal sensitivity. Ophthalmology. 2003;110:497-502.

12. Albietz JM, Lenton LM. Management of the ocular surface and tear film before, during, and after laser-in-situ keratomileusis. J Refract Surg. 2004;20:62-4.

13. Liu Z, Luo L, Zhang $Z$ et al. Tear film changes after phacoemulsification. Zhonghua Yan Ke Za Zhi. 2002;38:274- 6.

14. Ministry of Health and Family Welfare, Govt. Of India. National Programme for Control of Blindness, Annual Report, MOHFW. Govt of India 2010-11; 109-11.

15. Ram J, Gupta A, Brar GS, Kaushik S, Gupta A. Outcomes of phacoemulsification in patients with dry eye. J Cataract Refract Surg 2002; 28:1386-89.

16. Al-Aqaba MA, Fares U, Suleman H, Lowe J, Dua HS. Architecture and distribution of human corneal nerves. Br J Ophthalmol. 2010; 94:784-9.

17. Muller LJ, Vrensen GF, Pels L, Cardozo BN, Willekens B. Architecture of human corneal nerves. Invest Ophthalmol Vis Sci 1997; 38:985-94.

18. Sitompul R, Sancoyo GS, Hutauruk JA, Gondhowiardjo TD. Sensitivity change in cornea and tear layer due to incision difference on cataract surgery with either manual smallincision cataract surgery or phacoemulsification. Cornea 2008; (Suppl 1): S13-8.

19. Belmonte C, Acosta MC, Gallar J. Neural basis of sensation in intact and injured corneas. Exp Eye Res 2004; 78:513-25. 
20. Roberts CW, Elie ER. Dry eye symptoms following cataract surgery. Insight 2007; 32:14-21

21. Wilson WS, Duncan AJ, Jay JL. Effect of benzalkonium chloride on the stability of the precorneal tear film in rabbit and man. Br J Ophthalmol 1975; 59:667-9. 